\title{
Historias de vida y demografía de Physalaemus biligonigerus (Anura: Leptodactylidae) en una charca periurbana del centro de Argentina
}

\author{
Ana M. Nicolino ${ }^{1 *}$, Clarisa de L. Bionda ${ }^{1,2}$, Nancy E. Salas ${ }^{1} \&$ Adolfo L. Martino ${ }^{1}$ \\ 1. Laboratorio de Ecología, Facultad de Ciencias Exactas Físico-Químicas y Naturales, UNRC, Ruta Nacional No 36 km \\ 601, Río Cuarto, Córdoba, Argentina; amnicolino@gmail.com, cbionda@exa.unrc.edu.ar, \\ nsalas@exa.unrc.edu.ar, amartino@exa.unrc.edu.ar \\ 2. Consejo Nacional de Investigaciones Científicas y Técnicas (CONICET), Buenos Aires, Argentina. \\ * Correspondencia
}

Recibido 17-X-2017. Corregido 16-I-2018. Aceptado 13-II-2018.

\begin{abstract}
Demography and life histories of Physalaemus biligonigerus (Anura: Leptodactylidae) in a peri-urban pond in central Argentina. Knowledge of population dynamics provides information on the recent history, current status, and future trends of a population. Physalaemus biligonigerus is widely distributed in the Neotropics, this anuran has a high and explosive reproductive activity, but its populations seem to be composed of young individuals. The objective of this study was to analyze the population demography of $P$. biligonigerus in a semipermanent pond in Río Cuarto, Córdoba, Argentina and to carry out a population projection through 30 generations. The age structure was determined from the use of skeletochronology, and a static life table was later made. Fertility and survival rates were calculated for each stage, by which a Leslie Matrix was constructed and the population projection was performed. The projection shows oscillations in the number of individuals but in spite of this, the final tendency of the population is an increase in its size. The highest proportion of survivors occurs in the egg-to-larval stages and the mortality force acts intensively on the larval phase. The net reproductive rate indicates that the population of $P$. biligonigerus of the study pond is increasing. Rev. Biol. Trop. 66(2): 765-775. Epub 2018 June 01.
\end{abstract}

Key words: population demography; Physalaemus biligonigerus; age structure; life table; population projection.

Existe un interés creciente por parte de los biólogos de la conservación para conocer los efectos de la densidad sobre los rasgos de la historia de vida y la dinámica de las poblaciones (Forbes \& Calow, 2002; Vonesh \& De la Cruz, 2002; Henle, Sarre, \& Wiegand, 2004).

A partir de la determinación de la edad individual, es posible conocer la estructura de edad de una población, la cual provee información sobre su historia reciente, el estado actual y la probabilidad de comportamiento futuro de la misma (Dimmick \& Pelton, 1996). Algunos de los parametros poblacionales que influyen en los ciclos reproductivos, son la edad y tamaño a la maduración sexual, fecundidad, tasa de mortalidad, longevidad, crecimiento individual (Jofré, 2004).

El método de la esqueletocronología para la determinación de la edad y el crecimiento óseo es ampliamente utilizado en anfibios y está basado en el conteo de líneas o marcas de detención de crecimiento (Castanet \& Smirina, 1990; Sinsch, Di Tada, \& Martino, 2001; Sinsch, 2015). En especies de climas templados, el periodo cálido está representado por una banda ancha de crecimiento óseo; mientras que durante las estaciones más frías, se produce una disminución en el metabolismo y por lo tanto el cese del crecimiento óseo, en este caso se observa la formación de una línea en 
el periostio o LAG (line of arrested growth), que identifica a los periodos climáticamente desfavorables, este proceso que ocurre tanto en anfibios como en reptiles se denomina hibernación (Smirina, 1994). Si bien este proceso se dá en muchas especies de anfibios, existen excepciones en donde se marcan más de una línea o LAG por año (Iturra-Cid, Ortiz, \& Ibargüengoytía, 2010).

Physalaemus biligonigerus (Cope, 1861), es un anuro de tamaño pequeño (35 a 40 mm) (Cei, 1980; Gallardo, 1987) y de amplia distribución en Sudamérica. Presenta un comportamiento reproductivo explosivo, con una demografía efímera, con baja longevidad y compuesta la mayor parte de las poblaciones por individuos jóvenes (Martino, 1999).

Los antecedentes demográficos para la especie (Martino, 1999; Bionda, Lajmanovich, Salas, Martino, \& Di Tada, 2013) fueron realizados en poblaciones que habitaban agroecosistemas. Las proyecciones poblacionales realizadas por Bionda et al. (2013), resultaron desfavorables para gran parte de los sitios analizados para dicho estudio, prediciendo la extinción de las poblaciones al cabo de cierto número de generaciones. Debido a que es una especie que utiliza charcas temporarias para su reproducción, el éxito reproductivo y su permanencia depende de los años con clima favorable, mientras que los años de sequía perjudican enormemente a sus poblaciones (Martino, 1999). En este sentido, cabe mencionar que las charcas rodeadas de cultivos suelen presentar un hidroperíodo inestable ya sea porque son cuerpos de agua utilizados por el ganado o bien para riego (Bionda, Di Tada, \& Lajmanovich, 2011). Poco se conoce de dicha especie en otros tipos de ambientes, que permita saber si la estructura de edad observada por Martino (1999), compuesta la misma por individuos jóvenes, corresponde a una característica intrínseca de dicha especie o bien es respuesta a las características del hábitat donde vive.

El objetivo del presente trabajo es analizar la demografía poblacional de $P$. biligonigerus en una charca periurbana semipermanente ubicada dentro del campus de la Universidad Nacional de Río Cuarto.

\section{MATERIALES Y MÉTODOS}

Los muestreos a campo se realizaron desde diciembre 2014 a marzo 2015, en un cuerpo de agua semipermanente ubicado en el campus de la Universidad Nacional de Rio Cuarto, localmente denominado "Charca de las Brujas" (CH, 8 ha; Coordenadas: 330' $42^{\prime \prime} \mathrm{S}$, $64^{\circ} 18^{\prime} 10^{\prime \prime} \mathrm{O}, 428$ m.s.n.m.) la cual se abastece principalmente de escorrentías de aguas provenientes de desagües pluviales y no se realizan cultivos en un radio de $5 \mathrm{~km}$ (Bionda, 2011). Sobre la margen norte y en charcos temporarios predomina un ambiente de pastizal, mientras que en la margen sur dominan especies propias de la ecorregión del Espinal (De Angelo, 2003; Bionda, 2011). La región se caracteriza por presentar un clima semi-seco, con tendencia a semi-húmedo de las planicies, con pequeña deficiencia de agua, con invierno térmico de piedemonte y llanura (Capitanelli, 1979).

Se registraron las variables ambientales, $\mathrm{pH}$, temperatura del agua, conductividad, solutos disueltos totales y salinidad mediante un equipo digital 35-Series Tests TM Multiparámetro 35425-10. Además se obtuvieron las temperaturas y las precipitaciones mensuales desde 2007 hasta 2015, datos obtenidos en la estación meteorológica, la cual esta ubicada en las inmediaciones del campus de la Universidad Nacional de Río Cuarto, y facilitados por la Cátedra de Meteorología Agrícola.

Los individuos postmetamorficos fueron capturados utilizando trampas de caída viva (Corn, 1994), las cuales consistían en recipientes de plástico de $22 \mathrm{~cm}$ de diámetro y $40 \mathrm{~cm}$ de profundidad sin fondo, enterrados en el suelo. Las trampas se ubicaron en 3 transectas (4 trampas por transecta) en el borde norte de la charca (debido a la accesibilidad, y a que el borde sur es un bajo inundable), procurando abarcar toda su extensión. También se realizaron, muestreos utilizando la técnica de relevamiento por encuentros visuales (Crump \& Scott, 1994), tanto para la captura de individuos, como para 
la colecta y conteo de nidos. El sitio fue recorrido con una frecuencia de dos visitas semanales, preferentemente luego del registro de precipitaciones. Por cada individuo de $P$. biligonigerus capturado, se registró la clase de edad adulto o juvenil mediante la observación de caracteres sexuales secundarios, los cuales fueron identificados siguiendo a Bionda et al. (2013). También se determinó el sexo de los individuos, observando algunas características como la coloración y elasticidad del saco vocal, o presencia de huevos en las hembras. Se registró la longitud hocico-cloaca ( $\mathrm{LHC}, \mathrm{mm}$ ) con un calibre manual Vernier Somet Inox Extra (0.01 mm precisión) y la masa corporal $(\mathrm{P}, \mathrm{g})$ con una balanza Pesola ${ }^{\circledR}$ de 50/100/200 gramos. A cada individuo se les amputó el tercer dedo de la mano derecha, para luego estimar la edad de los mismos mediante la técnica de esqueletocronología y de esta forma también evitar el remuestreo (Bionda et al., 2011, 2013, 2015). Los individuos fueron liberados en el sitio de captura dos horas posteriores a la misma, previamente se les añadió un agente cicatrizante, antifúngico y antibacteriano, a la zona del corte para evitar futuras infecciones. Para la técnica de esqueletocronología se siguió el protocolo y la metodología detallada en Bionda et al. (2015). Teniendo el dato de la edad (el cual se trabaja como número de LAGs) y de los parámetros métricos, se calcularon las siguientes variables, siguiendo a Lescovar, Oromi, Sanuy y Sinsch (2006), Bionda et al. (2015) y Otero, Valetti, Bionda, Salas y Martino (2016): (1) Longevidad: el máximo número de LAGs contadas en individuos reproductivos; (2) Edad media de la población; (3) Edad de maduración o reproductiva: el mínimo numero de LAGs contados en los individuos reproductivos; (4) potencial reproductivo: diferencia entre la longevidad y la edad de maduración; (5) tamaño a la maduración: el promedio de LHC de los individuos a la edad de maduración; (6) Edad modal: moda en la distribución de edad; (7) tamaño corporal medio de la población: promedio de LHC en la población; (8) Tamaño corporal máximo de la población: máximo valor de LHC registrado en la población.

Se contabilizó el número de nidos en la charca y se colectaron 3 nidos, los cuales fueron transportados al laboratorio en recipientes separados para contar el número de huevos por nido, para esto, se separaron porciones pequeñas del nido y se contaron bajo la lupa, tratando de ejercer sobre los huevos la menor manipulación posible. Transcurridas 24 a 48 horas aproximadamente, se contó el número de larvas que eclosionaron por nido.

Tabla de vida: Se construyó una tabla de vida estática. Para ello, se calcularon las abundancias para cada estadío de la población. Se obtuvo el promedio de huevos por nido, y se lo multiplicó por el número de nidos observados en la charca durante los muestreos. Para el caso de las larvas, se utilizó la abundancia promedio obtenida de larvas eclosionadas por cada nido, y se multiplicó este valor por el número de nidos observados. Cabe aclarar que éste valor es sobreestimado dado que no todas las larvas eclosionadas de un nido sobreviven a lo largo de su metamorfosis. Pero este valor fue utilizado debido a que los muestreos de larvas a través del arrastre con un copo por la orilla de la charca, dio como resultado valores bajos en abundancia. No se obtuvieron datos de abundancia para juveniles en la temporada 2014-2015, por lo que se procedió a estimar la abundancia de los juveniles, teniendo en cuenta una mortalidad promedio en adultos de 0.45 , y por lo tanto una supervivencia de los mismos de 0.55 (Wells, 2007).

Las columnas que conformaron la tabla de vida fueron: número de individuos observados 
en cada fase o edad $(N)$, proporción de supervivencia $\left(l x=N_{r} / N_{n}\right)$, tasa de supervivencia

$$
\left(p_{x}=\frac{l_{x+1}}{l_{x}}\right),
$$

tasa de mortalidad $\left(q_{x}=1-p_{x}\right)$, esperanza de vida

$$
\left(e x=\sum_{x=0}^{x=i} \frac{l y}{l x}\right),
$$

fuerza o potencia de mortalidad $\left(K_{x}=\log 10 N_{x}\right.$ - $\left.\log 10 N_{x}+1\right)$, media de descendientes $(m x)$ y tasa media de reproducción

$$
\left(R_{0}=\sum_{x=0}^{x=i} l_{x} m_{x}\right)
$$

(Begon, Townsend, \& Harper, 1988).

Matriz de Leslie y proyección poblacional de $P$. biligonigerus: Se usó el método de KNM (Kiritani Nakasuki Manly) para estimar las tasas de supervivencia de cada estadío, siguiendo a Bécart, Aubry y Emmerson, 2007 y Bionda et al. (2013).

Con los datos de supervivencia por clase de edad y fecundidad, fue posible construir una matriz de Leslie (Leslie, 1945), la cual permite predecir la abundancia de cada clase de edad de un tiempo $t$ a un tiempo $t+1$. En este caso se construyó una proyección poblacional a 30 años. Utilizando el número de individuos de cada generación, se estimó también la tasa neta de reproducción $\mathrm{R}_{0}$ para cada año (Begon et al., 1988).

Tanto para el cálculo de los parámetros poblacionales, la matriz de Leslie y tabla de vida, se utilizó Microsoft Excel Office 2007. Para la comparación de parámetros poblacionales entre sexos se utilizó el test estadístico Mann Whitney, mientras que para la comparación de múltiples variables se utilizó el test de Kruskal-Wallis. Los analisis se realizaron a partir del programa STATISTICA 6.0 (Statsoft Inc., USA 2001).

\section{RESULTADOS}

Variables ambientales: En cuanto a las variables ambientales, para la temporada 20142015 , los valores medios obtenidos para las mismas fueron: temperatura del aire: $21.43 \pm 1.57$ ${ }^{\circ} \mathrm{C}$ (intervalo: $19.7-23.63{ }^{\circ} \mathrm{C}$ ), temperatura del agua: $26.73 \pm 5.95{ }^{\circ} \mathrm{C}$ (intervalo: $21.2-33.8{ }^{\circ} \mathrm{C}$ ), pH: $8.57 \pm 0.81$ (intervalo: 7.45-9.5), conductividad: $330.83 \pm 215.18$ (intervalo: 143-670), sales disueltas totales: $235.33 \pm 152.8$ (intervalo: 103-477), y salinidad: $161.48 \pm 104.35$ (intervalo: 73.8-328). Los valores más bajos de precipitación se dieron en las temporadas 2008-2009 y 2009-2010 (Fig. 1). Si bien, la precipitación

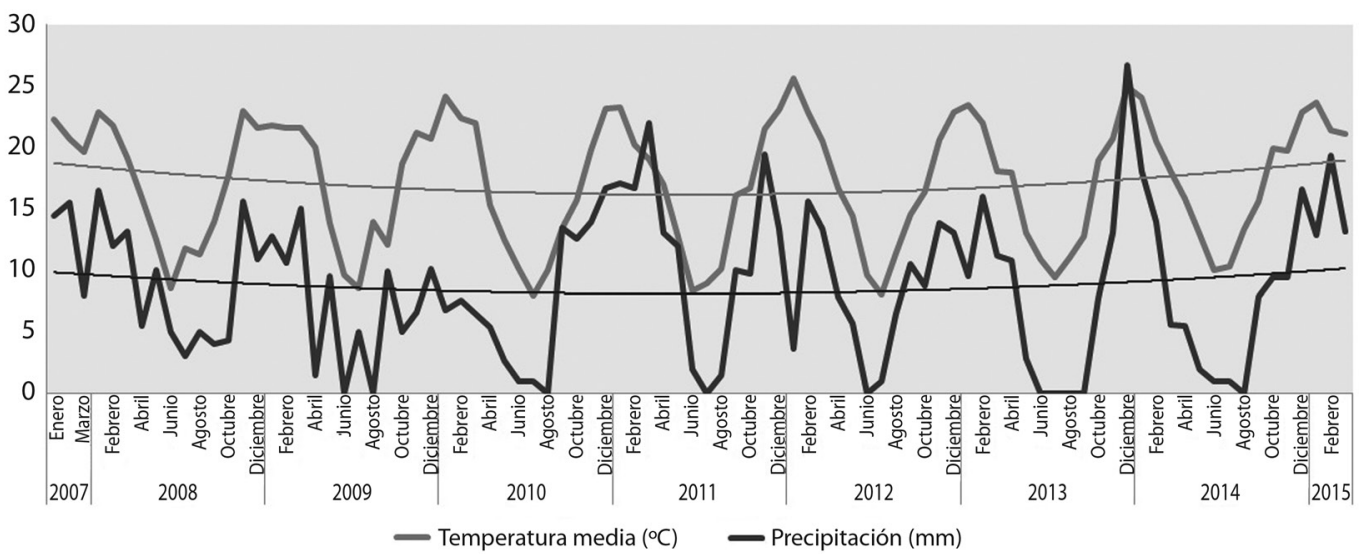

Fig. 1. Valores medios mensuales de las variables ambientales proporcionadas por la Cátedra de Meteorología Agrícola (UNRC): temperatura del aire y precipitación caída, desde enero del 2007 hasta marzo del 2015. Se grafican también las correspondientes líneas de tendencia.

Fig. 1. Monthly mean values of the environmental variables provided by the Chair of Agricultural Meteorology (UNRC): air temperature and falling precipitation, from January 2007 to March 2015. The corresponding trend lines are also plotted. 
CUADRO 1

Rasgos de historia de vida de P. biligonigerus

TABLE 1

Features life history of $P$. biligonigerus

\begin{tabular}{cccccccccc} 
Sexo & $\mathrm{N}$ & L.P & $\begin{array}{c}\text { Edad media } \\
(\text { LAGs })\end{array}$ & EM & ER & PR & $\begin{array}{c}\text { LHC a la } \\
\text { reproducción }\end{array}$ & LHC $(\mathrm{mm})$ & $\begin{array}{c}\text { LHC máximo } \\
(\mathrm{mm})\end{array}$ \\
$\mathrm{M}$ & 21 & $5(9.52 \%)$ & $3.48 \pm 0.81$ & $3(\mathrm{n}=9)$ & $2(9.52 \%)$ & 3 & $33.75 \pm 2.19$ & $34.69 \pm 2.44$ & 38.20 \\
$\mathrm{H}$ & 8 & $4(37.5 \%)$ & $3.37 \pm 0.52$ & $3(\mathrm{n}=5)$ & $3(62.5 \%)$ & 1 & $35.06 \pm 1.70$ & $35.27 \pm 2.54$ & 39.49 \\
\hline
\end{tabular}

L.P: longevidad poblacional (LAGs); EM: edad modal (LAGs); ER: edad a la reproducción (LAGs); PR: potencial reproductivo.

Life history traits of P. biligonigerus. L.P: population longevity (LAGs); MS: modal age (LAGs); ER: age at reproduction (LAGs); PR: reproductive potential.

anual caída entre 2007 y 2015, no muestra diferencias estadísticamente significativas (Kruskal-Wallis, $\mathrm{K}=9.36, \mathrm{P}=0.31$ ), la línea de tendencia muestra un leve crecimiento de los valores a lo largo de los años. En el caso de la temperatura, no se encontraron diferencias estadísticamente significativas entre los años (Kruskal-Wallis, $\mathrm{K}=4.77, \mathrm{P}=0.78$ ).

Estructura de edad: Se capturaron un total de 29 individuos adultos (machos: 21, hembras: 8). La esqueletocronología mostró que la longevidad máxima alcanzada para machos y hembras fue de 5 y 4 LAGs, $9.52 \%$ y $37.5 \%$ de la muestra total, respectivamente (Cuadro 1). Los resultados muestran que los machos se reproducen al menos a partir de las 2 LAGs y las hembras al menos a partir de 3 LAGs, lo cual se corresponde con el $9.52 \%$ y $62.5 \%$ de los individuos de la muestra, respectivamente. Así mismo, el intervalo de la edad encontrada en la muestra de las hembras varió solo entre 3 y 4 LAGs y en los machos varió entre 2 y 5 LAGs. El LHC al momento de la reproducción en los machos fue de $33.75 \pm 2.19$, $\mathrm{y}$ en las hembras fue de $35.06 \pm 1.70$, pero esta diferencia no resultó ser estadísticamente significativa (Mann-Whitney, $\mathrm{W}=3.0, \mathrm{P}=$ 0.56). Con respecto al potencial reproductivo, los machos registran al menos un potencial de 3 años mientras que las hembras al menos de1 año. La edad media en machos fue de $3.48 \pm 0.81$, que resultó algo mayor que en el caso de las hembras que fue $3.37 \pm 0.52$, aunque esta diferencia no fue estadísticamente significativa (Mann-Whitney, $\mathrm{W}=90.5, \mathrm{P}=0.75$ ). $\mathrm{El}$ tamaño medio de los machos fue de $34.69 \pm 2.44$ $\mathrm{y}$ de las hembras fue $35.27 \pm 2.54$, pero esta diferencia tampoco fue estadísticamente significativa (Mann-Whitney, $\mathrm{W}=76.5, \mathrm{P}=0.73$ ).

Tabla de vida: Se obtuvo un valor promedio de $1918.33 \pm 1473.78$ huevos por nido. En el caso de las larvas, se obtuvo en promedio 1 $192.66 \pm 686.95$ larvas eclosionadas por nido. El número de juveniles indicado en el cuadro 2 (n $=42$ ), se obtiene a partir de la mortalidad estimada por Wells (2007) de 0.45 desde el estadío de juveniles a adultos $(n=29)$. Cabe aclarar que fueron capturados sólo 2 adultos de 2 LAGs, menor número que los adultos de 3 LAGs, por lo tanto, tratándose de una tabla de vida estática y para evitar resultados negativos, los adultos de la clase 2 fueron calculados como el promedio entre los individuos juveniles y adultos de 3 LAGs (Cuadro 2).

La mayor proporción de supervivientes ocurre de huevos a larvas, y luego decrecen los valores bruscamente a partir de la fase de larvas a juveniles (Cuadro 2).

La fuerza de mortalidad (kx), actúa con mayor intensidad en la fase de larvas, seguida de los adultos de 4 LAGs. En las demás clases de edad este valor fue cercano a cero. La esperanza de vida (ex) muestra un decrecimiento en los valores desde la fase de juveniles a adultos, teniendo su pico máximo en la fase 
CUADRO 2

Tabla de vida estática de P. biligonigerus

TABLE 2

Static life table of $P$. biligonigerus

\begin{tabular}{cccccccccc}
$\mathrm{X}$ & $\mathrm{N}$ & $\mathrm{Lx}$ & $\mathrm{px}$ & $\mathrm{Qx}$ & $\mathrm{Ex}$ & $\mathrm{Kx}$ & $\mathrm{mx}$ & $1 x^{*} \mathrm{mx}$ \\
Hue & 19183 & 1.000 & 0.622 & 0.378 & 1.626 & 0.206 & 0 & 0 \\
Larv & 11927 & 0.622 & 0.003 & 0.997 & 1.007 & 2.453 & 0 & 0 \\
Juv & 42 & 0.002 & 0.357 & 0.642 & 2.000 & 0.447 & 0 & 0 \\
Ad 2 & 15 & 0.0008 & 0.933 & 0.066 & 2.800 & 0.030 & 0 & 0 \\
Ad 3 & 14 & 0.0007 & 0.786 & 0.214 & 1.928 & 0.105 & 959.16 & 0.700 \\
Ad 4 & 11 & 0.0006 & 0.182 & 0.818 & 1.182 & 0.740 & 959.16 & 0.550 \\
Ad 5 & 2 & 0.0001 & & 1.000 & 1.000 & & 959.16 & 0.100 \\
\hline
\end{tabular}

Tasa reproductiva neta: $\mathrm{R} 0=1.35$

$\mathrm{x}=$ clase de edad; $\mathrm{N}=$ número de individuos; $\mathrm{Lx}=$ proporción de supervivencia; $\mathrm{px}=$ tasa de supervivencia; $\mathrm{Q} \mathrm{x}=$ tasa de mortalidad; $\mathrm{Ex}=$ esperanza de vida; $\mathrm{Kx}=$ fuerza o potencia de mortalidad; $\mathrm{mx}=$ media de descendientes; Hue= huevos; Larv= larvas; Juv= juveniles; Ad 2= adultos con 2 LAGs; Ad 3= adultos con 3 LAGs; Ad 4= adultos con 4 LAGs; Ad 5= adultos con 5 LAGs.

Net reproductive rate: $\mathrm{RO}=\mathbf{1 . 3 5}$

$\mathrm{x}=$ age class $\mathrm{N}=$ number of individuals; $\mathrm{Lx}=$ survival proportion; $\mathrm{px}=$ survival rate; $\mathrm{Qx}=$ mortality rate; Ex $=$ life expectancy; $\mathrm{Kx}$ = force or power of mortality; $\mathrm{mx}=$ mean of descendants; Hue = eggs; Larv = larvae; Juv = juveniles; Ad 2 $=$ adults with 2 LAGs; Ad 3 = adults with 3 LAGs; Ad 4 = adults with 4 LAGs; Ad 5 = adults with 5 LAGs.

de juveniles. En los adultos reproductivos éste valor va disminuyendo gradualmente.

La tasa reproductiva neta $\mathrm{R}_{0}$ fue de 1.35 , lo cual indica que la población de P. biligonigerus está en incremento, teniendo en cuenta que cada individuo hembra de la población dejaría en promedio 1.35 individuos hembras como progenie.

Matriz de Leslie y proyección poblacional: Las tasas de supervivencia obtenidas mediante el método KNM, para cada estadío fueron: Huevos $=0.580$, Larvas $=0.013$ y Juveniles $=0.402$. En el cuadro 3, se muestra la matriz de Leslie construida con las tasas de supervivencia, el valor de fecundidad para la población y el vector etario utilizado inicialmente para las proyecciones poblacionales.

En la proyección poblacional para 30 generaciones (Fig. 2), se pueden observar oscilaciones en el tamaño poblacional, siendo

CUADRO 3

Matriz de Leslie y vector etario inicial para P. biligonigerus

TABLE 3

Matrix of Leslie and initial age vector for P. biligonigerus

\begin{tabular}{cccccc} 
S1 & S2 & S3 & F & Vector etario & Clases de edad \\
0 & 0 & 0 & 959.16 & 19183 & Huevos \\
0.580 & 0 & 0 & 0 & 11927 & Larvas \\
0 & 0.013 & 0 & 0 & 43 & Juveniles \\
0 & 0 & 0.402 & 0 & 29 & Adultos \\
\hline
\end{tabular}

$\mathrm{S} 1=$ tasa de supervivencia de huevos; $\mathrm{S} 2=$ tasa de supervivencia de larvas; $\mathrm{S} 3=$ tasa de supervivencia de juveniles; $\mathrm{F}=$ fecundidad.

$\mathrm{S} 1$ = egg survival rate; $\mathrm{S} 2$ = survival rate of larvae; $\mathrm{S} 3$ = survival rate of juveniles; $\mathrm{F}=$ fecundity. 


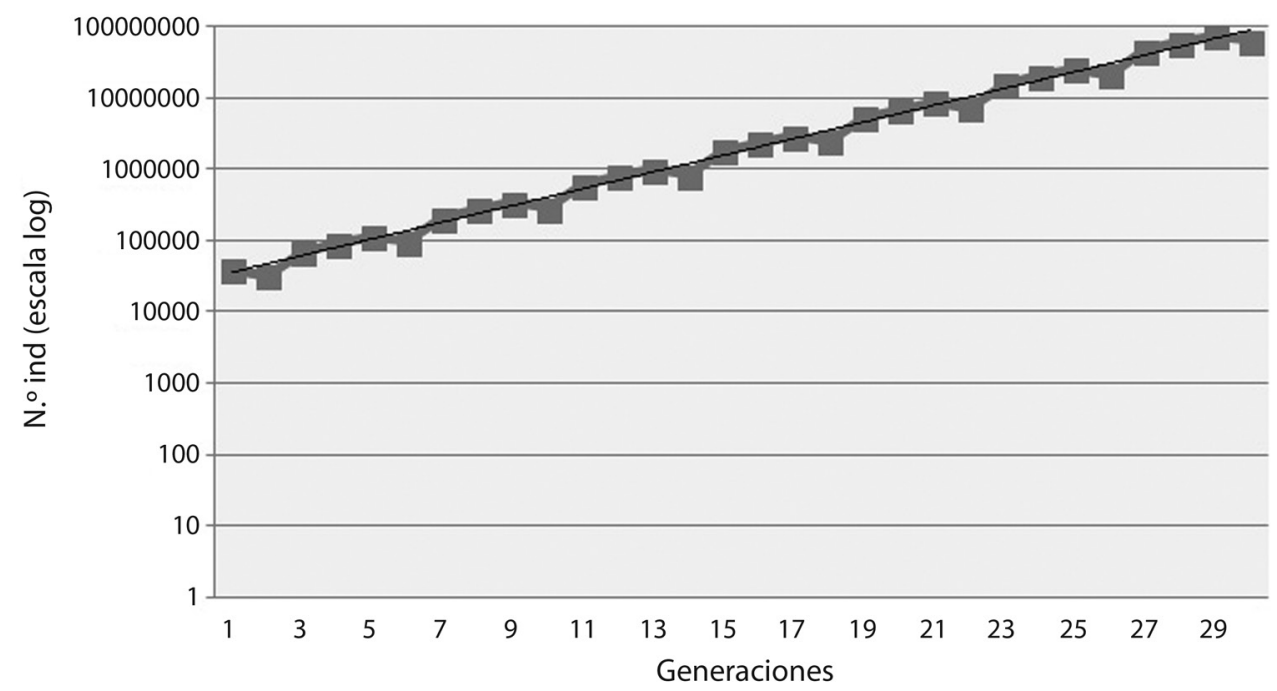

Fig. 2. Tendencia general de la proyección poblacional de $P$. biligonigerus para 30 generaciones. Número de individuos expresado en escala logarítmica.

Fig. 2. General trend of the population projection of $P$. biligonigerus for 30 generations. Number of individuals expressed in logarithmic scale.

la tendencia un aumento en el tamaño de la población en el tiempo.

\section{DISCUSIÓN}

La determinación de la fecundidad para una especie representa información valiosa no sólo para el conocimiento de la ecología y demografía, sino que también puede ser utilizado como un indicador para la determinación del status de conservación de las especies de anfibios anuros (ej. Lavilla et al., 2000). Sin embargo, en la bibliografía son escasos los trabajos que brindan este dato. En nuestro caso durante el año 2015, encontramos un valor promedio de huevos por nido de 1918.33 \pm 1473.78 ; mientras que Bionda et al. (2013), registró un número promedio de $822.19 \pm 94.72$ huevos por nido en $P$. biligonigerus en este mismo sitio para la temporada 2008-2009. El número medio de larvas eclosionadas en nuestro caso para el año 2015, fue de $1192.66 \pm 686.95$; mientras que Martino (1999), obtuvo un promedio de larvas de P. biligonigerus por nido de $2753.5 \pm$
1586.9 individuos. Este ultimo valor, varía con lo registrado en nuestro caso.

Para esta población, la longevidad encontrada (5 LAGs), resultó mayor que la registrada por Martino (1999) en otra población de la misma especie, en donde la mayoría de los individuos tenían entre 1 y 2 LAGs $(45.7 \%$ y $47.5 \%$ respectivamente) y un muy bajo porcentaje de éstos alcanzaron las 3 LAGs $(6.8 \%)$. Cabe aclarar que la población analizada por dicho autor, habita una zona con actividad agrícola intensiva, lo cual podría presuponer algún efecto sobre la población de este anuro en su longevidad. En este sentido, existe evidencia que en los agroecosistemas, las poblaciones de anfibios que allí habitan registran una edad media y tamaño corporal más bajo, con respecto a las poblaciones que habitan otros sitios menos alterados (Spear et al., 2009).

Determinados estudios sugieren que la maduración sexual en los machos ocurre a temprana edad comparada a las hembras (Liao, 2009; Liao \& Lu, 2010). Este retraso en la maduración de las hembras, les permitiría asignar dicha energía almacenada para el desarrollo y crecimiento en tamaño y de esta manera 
alcanzar la madurez sexual con una mejor condición corporal (Cummins, 1986; Marangoni, Barrasso, Cajade, \& Agostini, 2012). En nuestro caso, coincidimos con dichos autores e incluso a los mismos valores registrados por Marangoni et al. (2012) en P. fernandezae en donde las hembras se reprodujeron a partir de las 3 LAGs y los machos a partir de las 2 LAGs. Sin embargo, esta reproducción tardía en las hembras, sumada a una menor longevidad, implica que las hembras registren un bajo potencial reproductivo comparado a los machos. Al respecto, algunos autores (Smirina, 1994; Guarino, Tessa, Mercurio, \& Andreone, 2010; Oromi, Sanuy, \& Sinsch, 2012) consideran que una reproducción tardía estaría ligada a una mayor longevidad (trade-off entre ambos parámetros de historias de vida), sin embargo eso no es observado entre las hembras de la población estudiada aquí. De todas maneras, en las hembras una longevidad baja podría estar asociada al esfuerzo reproductivo de las mismas (Guarino, Di Già, \& Sindaco, 2008; Bionda et al., 2011). Este bajo potencial reproductivo en las hembras implica que las mismas presentan bajas expectativas reproductivas a lo largo de sus vidas (sólo un año) lo cual implica una característica que sí podría estar asociada a la demografía efímera, tal como lo plantea Martino (1999). Sin embargo, para alcanzar conclusiones más robustas al respecto, sería necesario un mayor número de hembras, y estudios en otras poblaciones de P. biligonigerus.

Los valores de supervivencia, ya sea los calculados por medio de la tabla de vida o la matriz de Leslie, indicaron el valor más bajo de esta variable para el estadío de larvas y la mayor supervivencia se da en el estadío de huevos, existiendo pequeñas variaciones entre ambos métodos usados. Cabe recordar, que estos valores pueden estar sobreestimados, dado que el número de larvas se obtuvo a partir del número de larvas eclosionadas de los nidos muestreados, y no de un muestreo sistemático en el cuerpo de agua, en donde existiría una menor probabilidad de que el número total de larvas eclosionadas sobrevivan al final de la metamorfosis. Del mismo modo, la mayor mortalidad se daría en el estadío de larvas y en los adultos de 4 LAGs. En este sentido, Bionda (2011) y Bionda et al. (2013), registraron mediante el método KNM para el mismo sitio de estudio, la menor tasa de supervivencia para el estadio de huevos. La fuerza de mortalidad actúa con mayor intensidad en el estadío de larvas. Mientras que para Bionda (2011) y Bionda et al. (2013), observaron que la mayor fuerza de mortalidad se dio en el estadío de huevos a larvas. Por lo que teniendo en cuenta nuestros datos y los mencionados en otros trabajos, la mortalidad actuaría con mayor fuerza en los estadios acuáticos, huevos y larvas. Esta es una característica observada con frecuencia en las poblaciones de $P$. biligonigerus, para este y otros sitios, ya que normalmente se observa una reproducción elevada, de allí que se considera una especie de reproducción explosiva y abundante, comparada a otras especies de la región de estudio, sin embrago, luego se advierte un reclutamiento bajo (Bionda et al., 2011, 2013). A pesar de ello, la tabla de vida arroja una tasa neta de reproducción mayor a 1 , lo cual indica que la población de $P$. biligonigerus de este sitio estaría en crecimiento.

La proyección poblacional, muestra oscilaciones del número de individuos a lo largo del tiempo, aunque la tendencia general es hacia un leve incremento de la población, lo cual coincide con los resultados obtenidos en la tabla de vida. Bionda (2011) y Bionda et al. (2013), realizaron una proyección poblacional de $P$. biligonigerus del año 2008 para este mismo sitio, encontrando un leve decrecimiento de la población a lo largo de las generaciones. Esta diferencia puede deberse a la variación en precipitaciones de los años de estudio, debido a que en nuestro caso (año 2015) las condiciones climáticas resultaron más favorables, con años previos de precipitaciones abundantes y temperaturas cálidas, mientras que los años previos al 2008, registraron escasas precipitaciones y temperaturas elevadas. Estas condiciones más favorables para 2015, podrían influir de manera en el hidroperíodo, calidad y cantidad de alimento, entre otros factores. Esto último puede ser una razón de las diferencias encontradas en 
la fecundidad de la población de $P$. biligonigerus en este trabajo (1 918.33 \pm 1473.78 huevos por nido) y la registrada por Bionda et al. (2013) (822.19 94.72 huevos para 2008-2009).

De acuerdo a la simulación obtenida podemos destacar que en los próximos 30 años, la población de $P$. biligonigerus del sitio Charca de las Brujas, se mantendrá estable con un leve incremento en el tiempo, en caso que las condiciones ambientales y demográficas se mantengan constantes a las registradas en este estudio. La población de $P$. biligonigerus analizada por Martino (1999), la cual estaba compuesta mayormente por individuos jóvenes, parece ser una característica de aquellas poblaciones que habitan ambientes alterados y probablemfignte de cuerpos de agua de hidroperíodo reducido, como suele suceder en los paisajes agrícolas. Por el contrario en la población analizada en este estudio la cual habita un ambiente poco alterado, ha indicado parámetros demográficos más favorables, con una longevidad mayor y un potencial reproductivo de varios años en el caso de los machos y algo menor en el caso de las hembras, lo cual podría estar asegurando la persistencia de la población. Se sugiere continuar con futuras investigaciones, que amplíen los estudios demográficos $\mathrm{y}$ de historias de vida, principalmente con un mayor número de hembras, así como estudios en otras poblaciones de la especie, que permitan aumentar el conocimiento de la dinámica poblacional para P. biligonigerus.

\section{AGRADECIMIENTOS}

Este trabajo fue financiado por la Agencia Nacional de Promoción Científica y Tecnológica (FONCyT) (BID-PICT 2012/0932) y SCyT UNRC (PPI 18/C448). Nuestro estudio fue autorizado por la Agencia Córdoba Ambiente (A.C.A.S.E.).

\section{RESUMEN}

El conocimiento de la dinámica de poblaciones provee información sobre la historia reciente, el estado actual y la tendencia futura de una población. Physalaemus biligonigerus es un anuro con una amplia distribución en el Neotrópico, presenta una actividad reproductiva elevada y explosiva, pero sus poblaciones parecen estar compuestas por individuos jóvenes. El objetivo de este estudio fue analizar la demografía poblacional de P. biligonigerus en una charca semipermanente en Río Cuarto, Córdoba, Argentina y realizar una proyección poblacional a 30 generaciones. Se determinó la estructura de edad utilizando esqueletocronología, y posteriormente se construyó una tabla de vida estática. Se calculo la fecundidad y las tasas de supervivencia para cada estadío con los que se construyó una Matriz de Leslie y se realizó la proyección poblacional. La proyección muestra oscilaciones en el número de individuos pero a pesar de esto, la tendencia final de la población es a un aumento en su tamaño. La mayor proporción de supervivientes se da en las fases de huevos a larvas, y la fuerza de mortalidad actúa con intensidad sobre la fase de larvas. La tasa neta reproductiva indica que la población de P. biligonigerus de la charca en estudio está en incremento.

Palabras clave: dinámica poblacional; Physalaemus biligonigerus; estructura de edad; tabla de vida; proyección poblacional.

\section{REFERENCIAS}

Bécart, E., Aubry, A., \& Emmerson, M. (2007). Monitoring the conservation status of natterjack toad (Bufo calamita) in Ireland, 2004 - 2006. Irish Wildlife Manuals, No. 31. Dublin, Ireland: National Parks and Wildlife Service, Department of the Environment, Heritage and Local, Government, Dublin, Ireland.

Begon, M., Townsend, C. R., \&. Harper, J. L. (1988). Ecología. Editorial Omega

Bionda, C. L. (2011). Dinámica poblacional de anfibios asociados a cultivos extensivos de la provincia de Córdoba, Argentina (Tesis de Doctorado), Universidad Nacional de Río Cuarto, Río Cuarto, Córdoba, Argentina.

Bionda, C. L., Di Tada, I. E., \& Lajmanovich, R. C. (2011). Composition of amphibian assemblages in agroecosystems from the central region of Argentina. Russian Journal of Herpetology, 18(2), 93-98.

Bionda, C. L., Kost, S., Salas, N. E., Lajmanovich, R. C., Sinsch, U., \& Martino, A. L. (2015). Age structure, growth and longevity in the common toad, Rhinella arenarum, from Argentina. Acta Herpetologica, 10(1), 55-62.

Bionda, C. L., Lajmanovich, R. C., Salas, N. E., Martino, A. L., \& Di Tada, I. E. (2013). Demografía poblacional de Rhinella arenarum (Anura: Bufonidae) y Physalaemus biligonigerus (Anura: Leiuperidae) en agroecosistemas de la provincia de Córdoba, Argentina. Revista de Biología Tropical, 6, 1389-1400. 
Capitanelli, R. G. (1979). III. Clima. En R. A.Vázquez, R. A. Miatello, \& Roqué, M. E. (Eds.), Geografia Física de la provincia de Córdoba (pp. 45-138). Buenos Aires: Ed. Boldt.

Castanet, J., \& Smirina, E. (1990). Introduction to the skeletochronological method in amphibians and reptiles. Annales des Sciences Naturelles, 13. ser. Zoologie, 11, 191-196.

Cei, J. M. (1980). Amphibians of Argentina. Monitore Zoologico Italiano. Monografia. 2, Nuova Serie, Firenze.

Corn, P. S. (1994). Straight-line drift fences and pitfall traps. In W. R. Heyer, M. A. Donnelly, R. W. McDiarmid, L. C. Hayek, \& M. S. Foster (Eds.), Measuring and monitoring biological diversity: Standard methods for amphibians (pp. 109-117). Washington: Smithsonian Institution press.

Crump, M. L. \& Scott, N. J. (1994). Standard techniques for inventory and monitoring. In W. R. Heyer, M. A. Donnelly, R. W. McDiarmid, L. C. Hayek, \& M. S. Foster (Eds.), Measuring and monitoring biological diversity: Standard methods for amphibians (pp. 75-142). Washington: Smithsonian Institution press.

Cummins, C. P. (1986). Temporal and spatial variation in egg size and fecundity in Rana temporaria. Journal of Animal Ecology, 55, 303-316.

De Angelo, C. D. (2003). Variación temporal de la actividad relativa de un ensamble de anuros (Trabajo Final de grado). Universidad Nacional de Río Cuarto, Argentina.

Dimmick, R. W., \& Pelton, M. R. (1996). Criteria of sex and age. In T. A. Bookhout (Ed.), Research and management techniques for wildlife and habitats ( $\mathrm{pp}$ 169-214). The Wildlife Society, Bethesda, Maryland.

Forbes, V. E., \& Calow, P. (2002). Population growth rate as a basis for ecological risk assessment of toxic chemicals. Philosophical Transactions of the Royal Society B, 357, 1299-1306.

Gallardo, J. M. (1987). Anfibios Argentinos. Guía para su Identificación. Buenos Aires: Biblioteca Mosaico, Librería Agropecuaria.

Guarino, F. M., Di Già, I., \& Sindaco, R. (2008). Age structure by skeletochronology in a declining population of Rana temporaria from northern Italy. Acta Zoologica Academiae Scientiarum Hungaricae, 54, 99-112.

Guarino, F. M., Tessa, G., Mercurio, V., \& Andreone, F. (2010). Rapid sexual maturity and short life span in the blue-legged frog and the rainbow frog from the arid Isalo Massif, southern-central Madagascar. Zoology, 113, 378-384.

Henle, K., Sarre, S., \& Wiegand, K. (2004). The role of density regulation in extinction processes and population viability analysis. Biodiversity and Conservation, 13, 9-52.

Iturra-Cid, M., Ortiz, J. C., \& Ibargüengoytía, N. R. (2010). Age, size, and growth of the Chilean frog Pleurodema thaul (Anura: Leiuperidae): latitudinal and altitudinal effects. Copeia, 2010(4), 609-617.

Jofré, G. M. (2004). Autoecología del sapo achaleño, Bufo achalensis CEI 1972 (Tesis doctoral). Universidad Nacional de Río Cuarto, Río Cuarto, Cordoba, Argentina.

Lavilla, E. O., Ponssa, M. L., Baldo, D., Basso, N., Bosso, A., Céspedez, J., Chebez, J. C., Faivovich, J., Ferrari, L., Lajmanovich, R., Langone, J. A., Peltzer, P., Úbeda, C., Vaira, M., \& Vera-Candioti, F. (2000). Categorización de los Anfibios de Argentina. In E. O. Lavilla, E. Richard, \& G. J. Scrocchi (Eds.), Categorización de los Anfibios y Reptiles de la República Argentina (pp. 11-34). Argentina: Asociación Herpetológica Argentina, San Miguel de Tucumán, Argentina.

Lescovar, C., Oromi, N., Sanuy, D., \& Sinsch, U. (2006). Demographic life history traits of reproductive natterjack toads (Bufo calamita) vary between northern and southern latitudes. Amphibia-Reptilia, 27, 365-375.

Leslie, P. H. (1945). On the use of matrices in certain population mathematics. Biometrika, 35, 183-212.

Liao, W. B. (2009). Elevational variation in the life-history of anurans in a subtropics montane forest of Sichuan, southwestern China (Ph.D thesis). Wuhan University, Wuhan, China.

Liao, W. B., \& Lu, X. (2010). Age and growth of a subtropical high-elevation torrent frog, Amolops mantzorum, in western China. Journal of Herpetology, $44,172-176$.

Marangoni, F., Barrasso, D. A., Cajade, R., \& Agostini, G. (2012). Body size, age and growth pattern of Physalaemus fernandezae (Anura: Leiuperidae) of Argentina. North-Western Journal of Zoology, 8(1), 63-71.

Martino, A. L. (1999). Análisis estructural de una comunidad de anfibios (Barreto, Córdoba, Argentina) (Tesis Doctoral). Universidad Nacional de Río Cuarto, Río Cuarto, Córdoba, Argentina.

Oromi, N., Sanuy, D., \& Sinsch, U. (2012). Altitudinal variation of demographic life-history traits does not mimic latitudinal variation in natterjack toads (Bufo calamita). Zoology, 115, 30-37.

Otero, M. A., Valetti, J. A., Bionda, C. L., Salas, N. E., \& Martino, A. L. (2016). Are ploidy and age size-related? A comparative study on tetraploid Pleurodema Kriegi and octoploid P. cordobae (Anura: Leptodactylidae) from Central Argentina. Zoologischer Anzeiger. DOI:10.1016/j.jcz.2016.07.005 
Sinsch, U. (2015). Review: Skeletochronological assessment of demographic life-history traits in amphibians. The Herpetological Journal, 25(1), 5-13.

Sinsch, U., Di Tada, I. E., \& Martino, A. L. (2001). Longevity, demography and sex-specific growth of the Pampa de Achala toad, Bufo achalensis CEI, 1972. Studies on Neotropical Fauna and Environment, 36, 95-104.

Smirina, E. M. (1994). Age determination and longevity in amphibians. Gerontology, 40, 133-146.

Spear, P. A., Boily, M., Giroux, I., Deblois, C., Leclair, M. H., Levasseur, M., \& Leclair, R. (2009). Study design, water quality, morphometrics and age of the bullfrog, Rana catesbeiana, in sub-watersheds of the Yamaska River drainage basin, Québec, Canada. Aquatic Toxicology, 91, 110-117.

Statsoft. (2001). Statistica for Windows, Release 6.0. Computer program manual. Tulsa, OK, USA: Statsoft, Inc.

Vonesh, J. R., \& De la Cruz, O. (2002). Complex life cycles and density dependence: assessing the contribution of egg mortality to amphibian declines. Oecologia, 133, 325-333.

Wells, K. D. (2007). The ecology and behavior of amphibians. Illinois, EE.UU: Universidad de Chicago, Chicago. 